\title{
A bilingual dietary intervention early in treatment is feasible and prevents weight gain in childhood acute lymphoblastic leukemia (ALL)
}

\author{
Michelle Walters ${ }^{1}$, Catriona Mowbray ${ }^{2}$, Tracey Jubelirer ${ }^{3}$, Shana Jacobs ${ }^{4}$, Kara Kelly ${ }^{5}$, \\ Karen Smith $^{3}$, Yujing Yao ${ }^{6}$, Jin Zhezhen ${ }^{7}$, and Elena Ladas ${ }^{6}$ \\ ${ }^{1}$ Columbia University Medical Center \\ ${ }^{2}$ Children's National Medical Center \\ ${ }^{3}$ The Children's Hospital of Philadelphia \\ ${ }^{4}$ Children's National Health System \\ ${ }^{5}$ Roswell Park Cancer Institute \\ ${ }^{6}$ Columbia University \\ ${ }^{7}$ Columbia University Mailman School of Public Health
}

October 15, 2020

\begin{abstract}
Background: Childhood acute lymphoblastic leukemia (ALL) is the most common pediatric malignancy. The onset of obesity during childhood ALL has been well established and is associated with inferior survival rates and increased treatment-related toxicities. This pilot study sought to determine if a dietary intervention is feasible and minimizes weight gain during the initial phases of treatment for ALL. Methods: Participants were recruited from four institutions, fluent in English or Spanish, between 5-21 years old, and enrolled within three days of starting induction therapy. Participants were counseled for six months to follow a low glycemic diet. Dietary and anthropometric data were collected at baseline, end of induction, and end of month six (NCT03157323). Results: Twenty-three of 28 participants $(82.1 \%)$ were evaluable and included in the analysis. Dietary intake of several nutrients targeted by the nutrition intervention declined (sugar, $\mathrm{P}=0.003$ and glycemic load, $\mathrm{P}=$ 0.053). We also observed a persistent increase in total vegetables across each timepoint $(\mathrm{P}=0.015)$ and by the end of the intervention $(\mathrm{P}=0.033)$. Importantly, we did not observe an increase in body mass index z-score during induction or over the six-month intervention period. Most families found the nutrition intervention easy to follow (60\%) and affordable (95\%) despite simultaneous initiation of treatment for ALL. Conclusions: A six-month nutrition intervention initiated during the initial phase of treatment for childhood ALL is feasible and may prevent weight gain. Our preliminary findings need to be confirmed in a larger clinical trial.
\end{abstract}

\section{Study Design and Procedures}

This is a multi-center pilot study consisting of a six-month dietary intervention. Participants were recruited from Children's Hospital of Philadelphia (Philadelphia, PA), Children's National Hospital (Washington, DC), Roswell Park Comprehensive Cancer Center (Buffalo, NY), and Columbia University Irving Medical Center (New York, NY) from June 2017 through August 2019. Dietary intake and anthropometric measurements were obtained at study entry (diagnosis), end of induction (approximately 30 days from initiation of treatment), and end of month six of treatment. The study was approved by the institutional review boards at all participating centers and was performed in accordance with the Helsinki Declaration. The trial was registered with clinicaltrials.gov (NCT03157323). 


\section{Participants}

Eligible participants were between 5-21 years old with newly diagnosed ALL (B- or T-cell) and fluent in English or Spanish. All participants were enrolled within three days of starting induction therapy. Consent and assent, when applicable, were obtained from parents and participants. Participants were removed from the study if they relapsed, deceased, or left the institution.

\section{Nutrition Intervention}

Participants were provided the option to initiate nutrition counseling at diagnosis or during induction. Dietitians or trained study staff at each institution counseled participants on following a low glycemic index (GI) diet at weekly intervals for three months (intervention phase) and then at monthly intervals for the next three months (maintenance phase). Counseling sessions were conducted in person during routine clinic visits or hospital admission or via telemedicine. A low GI diet was selected due to the data supporting its efficacy in weight management in both adult and pediatric settings. ${ }^{12}$ Briefly, GI describes how a controlled portion of food affects postprandial rise in blood glucose; high GI foods result in faster and higher increases in blood glucose compared to low GI foods. Glycemic load (GL) accounts for the GI and quantity of available carbohydrate in the food. The intervention followed the Traffic Light approach, which has been utilized successfully in counseling children and adolescents. ${ }^{13}$ Standardized nutrition education materials were provided to each collaborating institution. Hospital and cafeteria menus were also categorized according to the Traffic Light approach. Participants and their families received written education materials in English or Spanish to support the counseling sessions, which provided instruction on grocery shopping and dining out. Caloric restriction was not emphasized due to research supporting the satiety effects of a low GI diet. ${ }^{14}$

\section{Data Collection}

Dietary intake was measured by two 24-hour diet recalls collected within a 7-day period at each timepoint to account for variation in intake. Dietary output was averaged across the two surveys as previously validated. ${ }^{15}$ Dietary recalls were administered in either English or Spanish using the Automated Self-Administered 24hour (ASA24) Dietary Assessment Tool, version 2016, developed by the National Cancer Institute (Bethesda, MD) ${ }^{16}$ The ASA24 system allowed the participant or their parent to self-administer 24-hour diet recalls using a web-based platform. ASA24 utilizes the United States Department of Agriculture's Food and Nutrient Database for Dietary Studies (FNDDS) food codes and nutrient values. The method previously described by Lin et $\mathrm{al}^{17}$ was used to assign GI values. Briefly, utilizing the international tables of glycemic index and load, 2008, ${ }^{18}$ we searched the I-tables $2008 \mathrm{~A} 1$, and secondarily, I-tables $2008 \mathrm{~A} 2$, for identical or closely matched foods based on their nutritional profiles and preparation methods. If no similar food existed, we calculated GI values by summing the weighted GI value of the component foods. Recipes for combination foods were pulled from FNDDS ingredients lists. ${ }^{19}$

Anthropometric measures including height and weight were obtained during routine clinic visits. Body mass index (BMI) values were calculated from weight and height (in $\mathrm{kg} / \mathrm{m}^{2}$ ), and BMI percentile and $z$-scores were calculated according to the 2000 Centers for Disease Control and Prevention reference norms. ${ }^{20}$ Children and adolescents $<20$ years old were classified as underweight, normal weight, overweight, or obese based on BMI-for-age percentile values $<5$ th, 5-84th, 85-94th, and [?] 95 th percentile, respectively. Participants $>20$ years were classified utilizing adult classifications and then converted to z-scores.

A one-time Ease of Study Survey was collected following the completion of the nutrition intervention. The six-question survey was constructed using the five-point Likert scale and queried participants about their experience with the dietary intervention. ${ }^{21}$ Parent surveys were completed if the participant was $<18$ years old and patient surveys were completed if the participant was [?]18 years old.

\section{Statistical Analysis}

Sample size was determined by the accrual time period. Due to our relatively small sample size $(\mathrm{N}=23)$, robust nonparametric statistical methods were used for data analysis. The continuous variables were summarized by median, minimum, and maximum and the categorical variables were summarized by frequency 
and rates. At each visit timepoint, boxplot was used to illustrate the distribution of the median BMI z-score overall and within subgroups. The Friedman's test was used to examine if there is any significant difference in median BMI z-score across the three timepoints, the Fisher's exact test was used to examine the difference in BMI categories for the three visits, and the Wilcoxon rank sum test was used for comparison of BMI z-score between groups at each visit time point. The Friedman's test was also used to examine the change of dietary intake and diet quality over time. The Wilcoxon signed rank test was performed to investigate the change of dietary intake and diet quality between study entry and end of month six. Median regression analysis was used to evaluate the relationship between change in dietary factors and change in BMI z-score from study entry to end of induction or end of month six after adjustment of age, gender, ethnicity, and BMI z-score at study of entry. The R 4.0 and SAS 9.4 (Cary, NC) were used for data analysis.

\section{RESULTS}

Twenty-eight participants enrolled in the study between June 2017 through August 2019. Two participants withdrew due to feeling too overwhelmed by the diagnosis of ALL and three withdrew due to reluctance to complete study assessments; all withdrew within the first two months. Two additional participants were removed from the study prior to end of month six due to change in institution and clinical course, and thus did not receive the full intervention. The analysis represents $23(82.1 \%)$ evaluable participants.

The demographic and clinical characteristics are presented in Table 1 . The cohort reflected the demographics of childhood ALL with slightly more males $(52.2 \%)$ than females $(47.8 \%) ; 43.5 \%$ were Hispanic and $52.2 \%$ were white. Nearly $22 \%$ of participants were classified as obese at study entry; higher than previously reported studies in ALL. ${ }^{6,22}$ Most participants (82.6\%) selected to initiate nutrition counseling at study entry. Of the four participants who elected to initiate nutrition counseling $(\mathrm{N}=4)$ later in the induction phase of treatment, the majority were female (75\%), Non-Hispanic and white (75\%), and younger (less than 10 years old) $(75 \%)$.

\section{Dietary Intake}

Table 2 presents dietary data by timepoint. We observed a statistically significant difference in median calorie intake across all three timepoints for the entire cohort $(P=0.013)$; however, by end of month six, values returned to baseline $(P=0.250)$. For macronutrients, carbohydrate intake decreased or stabilized by 6-months $(P=0.035, P=0.064$, respectively). Additionally, we observed a corresponding significant increase in protein from study entry to end of month six $(P=0.027)$ likely owing to the underlying tenets of the low-glycemic diet. Despite increases in calorie intake, we observed a significant reduction in the intake of sugar, a nutrient strongly associated with weight gain, across all three timepoints at the end of induction $(P=0.0051)$ and 6 -months $(P=0.003)$. We also observed a non-significant increase in fiber. No difference in median GI was observed throughout the intervention $(P=0.920)$; however, a notable change in energy-adjusted GL was observed $(P=0.030)$ and there was a trend towards a sustained reduction in energy-adjusted GL from study entry to end of month six $(P=0.053)$.

Several aspects of diet quality improved over the study period (Supplementary Table \#S1). Total vegetable intake increased and remained elevated at the end of month six compared to intakes at study entry $(P=$ 0.033). Intake of added sugars decreased by $54.5 \%$ from study entry to end of month six $(P=0.021)$ and an increased intake of starchy vegetables excluding white potatoes $(P=0.033)$ was observed. Other notable changes in diet quality across the intervention included increases in total protein foods, total grains, and oils ( $P=0.006, P=0.004$, and $P=0.009$, respectively), though differences were not sustained by the end of month six $(P=0.130, P=0.140$, and $P=0.280$, respectively $)$.

Body Mass Index

The distribution of median BMI z-score is presented in Figure 1. We did not observe any significant difference in BMI z-score over the first six months of treatment (Fig. 1A) or significant changes in weight classification across the study period (Supplementary Table \#S2). To evaluate differences in BMI by clinical and demographic variables associated with increased BMI, Wilcoxon rank sum test was performed and evaluated BMI 
by risk group, sex, and ethnicity at each timepoint (Fig. 1B-D). BMI z-scores were not significantly different between low risk, intermediate risk, and high risk groups at study entry, end of induction, or end of 6-months (Fig. 1B, $P=0.89, P=0.48$, and $P=0.19$, respectively). BMI z-scores were also not significantly different between females and males at each timepoint (Fig. 1C, $P=0.69, P=0.24$, and $P=0.94$, respectively) or Hispanics and Non-Hispanics at each timepoint (Fig. 1D, $P=0.71, P=0.88$, and $P=0.13$, respectively).

We performed median regression analysis to evaluate the relationship between changes in dietary variables and BMI z-score (Supplementary Table \#S3). We did not find any additional significant relationships between dietary intake of specific nutrients or food groups and BMI z-score after adjustment for age at study entry, sex, ethnicity, and baseline BMI z-score.

\section{Feasibility}

Twenty participants $(87 \%)$ completed the End of Study Survey. Sixty percent of participants agreed that the diet was easy to follow during treatment and believed that they were successful at doing so. Further, $95 \%$ of participants found the diet affordable and $90 \%$ agreed it was easy to understand. Eighty-five percent of participants planned to continue the diet after the intervention ended.

\section{DISCUSSION}

To our knowledge, our results are the first to suggest that a dietary intervention initiated during the initial phases of treatment for childhood ALL is feasible, and may improve diet quality and stabilize weight. Despite an observed increase in calories, potentially due to administration of steroids, participants were able to improve dietary quality through increased intake of vegetables and decreased intake of added sugars. With the sustainability and long-term efficacy of calorie-restricted diets being questioned, emphasizing the quality over the quantity of calories consumed is becoming increasingly important in interventions targeting weight management. ${ }^{23,24}$ This finding is especially important in light of a recent article highlighting the importance of diet quality, rather than calorie counting, in reducing acute toxicities in childhood ALL. ${ }^{25}$

Few published studies have investigated a nutrition intervention during treatment for childhood ALL. All of the existing studies have initiated the nutrition intervention during the maintenance phase of therapy, which is after the initial onset of obesity. ${ }^{26-29}$ Our intervention was not designed to be a weight-loss intervention. The nutrition education focused on dietary quality and avoidance of excessive consumption of obesitypromoting foods. Thus, an important finding of our study is the reduction of obesity-promoting foods and nutrients, mostly notably added sugar. ${ }^{30,31}$ This builds upon our previous work that found an increased intake from carbohydrates, more so than total calories, predicted higher BMI at continuation. ${ }^{22}$ Aligned with the dietary principles relied upon in this study, increased dietary fiber intake through consumption of fruit, vegetables, whole grains, and legumes is a well-established strategy to prevent and treat childhood obesity. ${ }^{32}$ We observed a notable increase in intake of total vegetables over the study period. Though we did not find a significant change in GI values throughout the study, we did observe significant reductions in GL. The GL reflects the quantity and quality of carbohydrate and has been found to be a better indicator of glycemic response compared to GI. ${ }^{33}$ Taken together, our results emphasize the need to focus on dietary quality versus traditional calorie counting approaches. Additional research is warranted.

The available literature examining weight gain during childhood ALL treatment consistently reports excessive weight gain during the induction and maintenance phases of therapy ${ }^{6,7,34,35}$ Previous studies also have found that weight gain during induction predicts weight gain in the later phases of treatment, which further underscores the importance of the findings of our intervention. In contrast to the existing literature, our analyses did not reveal a significant increase in BMI early on treatment. However, our findings must be interpreted considering the pilot nature of the study.

There are several strengths of our study. This was a standardized nutrition intervention provided at multiple centers with an ethnically diverse population. A standardized dietary approach was implemented and was able to be personalized based on variation in regional location, ethnicity, and socioeconomic status. Rather than focusing on calorie counting or weight reduction, our intervention focused on improving dietary quality 
within the sociodemographic variables of the participant, which made the intervention easy to follow. A dietitian was not always the staff member to provide dietary counseling due to understaffing at certain sites, demonstrating that the intervention can be delivered especially with the expansion of telehealth. The high percentage of participants who reported the diet to be easy to follow and economical suggests this study could be replicated and received with interest in a larger, multi-center setting.

Our results should be interpreted in light of limitations. Dietary intake was self-reported; therefore, we cannot exclude the possibility of misreporting. ${ }^{36}$ However, this was minimized by the collection of two 24hour recalls within one week of each other. We did not collect information on physical activity, although we believe that this is a small effect as most patients are sedentary during the initial, most intensive, phases of treatment for childhood ALL. Socioeconomic status was not considered in this analysis, but will be included in subsequent studies. ${ }^{37}$ Finally, despite similar approaches for childhood ALL treatment, participants were diagnosed with either B- or T-cell ALL and treated on COG and DFCI protocols, both of which consist of varying doses and duration of steroids. While our limited sample size precluded a comprehensive analysis of these features, our results suggest that this intervention is feasible regardless of treatment regimen, risk group, or ALL phenotype, further improving its generalizability in a larger, multi-center setting.

This study demonstrates that a six-month nutrition intervention initiated in the earliest phase of treatment for childhood ALL is feasible and well-received. Preliminary analysis indicates that our dietary intervention may prevent the excessive weight gain typically seen during the initial phases of treatment. Larger lifestyle intervention studies focused on early prevention of weight gain should be prioritized. Efforts to confirm our findings in a larger, randomized controlled trial within several cooperative groups settings are underway.

Conflict of interest : None of the authors have any conflict of interest to report.

\section{Author contributions:}

Michelle Walters: Project administration, data curation, formal analysis, writing - original draft, and writing - review and editing. Catriona Mowbray: Project administration, data curation, formal analysis, writing original draft, and writing - review and editing. Tracey Jubelirer: Conceptualization, methodology, project administration, data curation, formal analysis, writing - original draft, and writing - review and editing. Shana Jacobs: Conceptualization, methodology, project administration, data curation, formal analysis, writing - original draft, and writing - review and editing. Kara Kelly: Conceptualization, methodology, project administration, data curation, formal analysis, writing - original draft, and writing - review and editing. Karen Smith: Project administration, data curation, formal analysis, writing - original draft, and writing review and editing. Yujing Yao: Data curation, formal analysis, writing - original draft, and writing - review and editing. Zhezhen Jin: Data curation, formal analysis, writing - original draft, and writing - review and editing. Elena Ladas: Conceptualization, methodology, project administration, data curation, formal analysis, writing - original draft, and writing - review and editing, supervision.

Acknowledgments : We thank the patients, families, physicians, nurses, nutritionists, research coordinators, and all others who participated in the data collection for this clinical trial.

Data availability statement: Data is available from the corresponding author upon reasonable request.

Funding: American Cancer Society, Mentored Research Scholar Grant, 127000-MRSG-14-157-01-CCE (E Ladas), Gabrielle's Angels Foundation, No 099 (E Ladas).

\section{REFERENCES}

1. Ward E, DeSantis C, Robbins A, Kohler B, Jemal A. Childhood and adolescent cancer statistics, 2014. CA Cancer J Clin . 2014;64:83-103.

2. Hunger SP, Loh ML, Whitlock JA, et al. Children's Oncology Group's 2013 blueprint for research: Acute lymphoblastic leukemia. Pediatr Blood Cancer . 2013;60:957-963. 
3. Orgel E, Genkinger JM, Aggarwal D, Sung L, Nieder M, Ladas EJ. Association of body mass index and survival in pediatric leukemia: A meta-analysis. Am J Clin Nutr . 2016;103:808-817.

4. Orgel E, Sposto R, Malvar J, et al. Impact on survival and toxicity by duration of weight extremes during treatment for pediatric acute lymphoblastic leukemia: A report from the Children's Oncology Group.J Clin Oncol . 2014;32:1331-1337.

5. Meenan CK, Kelly JA, Wang L, Ritchey AK, Maurer SH. Obesity in pediatric patients with acute lymphoblastic leukemia increases the risk of adverse events during pre-maintenance chemotherapy. Pediatr Blood Cancer . 2019;66:e27515.

6. Withycombe JS, Smith LM, Meza JL, et al. Weight change during childhood acute lymphoblastic leukemia induction therapy predicts obesity: A report from the Children's Oncology Group. Pediatr Blood Cancer . 2015;62:434-439.

7. Esbenshade AJ, Simmons JH, Koyama T, Koehler E, Whitlock JA, Friedman DL. Body mass index and blood pressure changes over the course of treatment of pediatric acute lymphoblastic leukemia. Pediatr Blood Cancer . 2011;56:372-378.

8. Zhang FF, Liu S, Chung M, Kelly MJ. Growth patterns during and after treatment in patients with pediatric ALL: A meta-analysis. Pediatr Blood Cancer . 2015;62:1452-1460.

9. Kourti M, Tragiannidis A, Makedou A, Papageorgiou T, Rousso I, Athanassiadou F. Metabolic syndrome in children and adolescents with acute lymphoblastic leukemia after the completion of chemotherapy.J Pediatr Hematol Oncol . 2005;27:499-501.

10. Oeffinger KC. Are survivors of acute lymphoblastic leukemia (ALL) at increased risk of cardiovascular disease? Pediatr Blood Cancer . 2008;50:462-467.

11. Wang KW, Ladhani S, Empringham B, et al. Bariatric interventions in obesity treatment and prevention in pediatric acute lymphoblastic leukemia: A systematic review and meta-analysis. Cancer Metastasis Rev . 2020;39:79-90.

12. Barclay AW, Petocz P, McMillan-Price J, et al. Glycemic index, glycemic load, and chronic disease risk-a meta-analysis of observational studies. Am J Clin Nutr . 2008;87:627-637.

13. Epstein LH, Valoski A, Wing RR, McCurley J. Ten-year outcomes of behavioral family-based treatment for childhood obesity. Health Psychol . 1994;13:373-383.

14. Warren JM, Henry CJ, Simonite V. Low glycemic index breakfasts and reduced food intake in preadolescent children. Pediatrics . 2003;112:e414.

15. Crispim SP, de Vries JH, Geelen A, et al. Two non-consecutive $24 \mathrm{~h}$ recalls using EPIC-Soft software are sufficiently valid for comparing protein and potassium intake between five European centres-results from the European food consumption validation (EFCOVAL) study. Br J Nutr . 2011;105:447-458.

16. Subar AF, Kirkpatrick SI, Mittl B, et al. The Automated Self-Administered 24-hour dietary recall (ASA24): A resource for researchers, clinicians, and educators from the National Cancer Institute. J Acad Nutr Diet . 2012;112:1134-1137.

17. Lin CS, Kimokoti RW, Brown LS, Kaye EA, Nunn ME, Millen BE. Methodology for adding glycemic index to the National Health and Nutrition Examination Survey nutrient database. J Acad Nutr Diet . 2012;112:1843-1851.

18. Atkinson FS, Foster-Powell K, Brand-Miller JC. International tables of glycemic index and glycemic load values: 2008. Diabetes Care . 2008;31:2281-2283.

19. U.S. Department of Agriculture. USDA food and nutrient database for dietary studies 2011-2012. http://www.ars.usda.gov/ba/bhnrc/fsrg. 
20. Kuczmarski RJ, Ogden CL, Guo SS, et al. 2000 CDC growth charts for the United States: Methods and development. Vital Health Stat 11 . 2002:1-190.

21. Young PC, West SA, Ortiz K, Carlson J. A pilot study to determine the feasibility of the low glycemic index diet as a treatment for overweight children in primary care practice. Ambul Pediatr . 2004;4:28-33.

22. Ladas EJ, Orjuela M, Stevenson K, et al. Fluctuations in dietary intake during treatment for childhood leukemia: A report from the DALLT cohort. Clin Nutr . 2019;38:2866-2874.

23. Ludwig DS, Ebbeling CB. The carbohydrate-insulin model of obesity: Beyond "calories in, calories out". JAMA Intern Med . 2018;178:1098-1103.

24. Bailes JR, Strow MT, Werthammer J, McGinnis RA, Elitsur Y. Effect of low-carbohydrate, unlimited calorie diet on the treatment of childhood obesity: A prospective controlled study. Metab Syndr Relat Disord . 2003;1:221-225.

25. Ladas EJ, Blonquist TM, Puligandla M, et al. Protective effects of dietary intake of antioxidants and treatment-related toxicity in childhood leukemia: A report from the DALLT cohort. J Clin Oncol . 2020;38:2151-2159.

26. Li R, Donnella H, Knouse P, et al. A randomized nutrition counseling intervention in pediatric leukemia patients receiving steroids results in reduced caloric intake. Pediatr Blood Cancer . 2017;64:374-380.

27. Hill R, Hamby T, Bashore L, et al. Early nutrition intervention attenuates weight gain for pediatric acute lymphoblastic leukemia patients in maintenance therapy. J Pediatr Hematol Oncol . 2018;40:104-110.

28. Wright MJ, Collins L, Christie A, Birken K, Dettmer E, Nathan PC. A comprehensive healthy lifestyle program for children receiving treatment for acute lymphoblastic leukemia: Feasibility and preliminary efficacy data. Rehabil Oncol . 2013;31:6-13.

29. Moyer-Mileur LJ, Ransdell L, Bruggers CS. Fitness of children with standard-risk acute lymphoblastic leukemia during maintenance therapy: Response to a home-based exercise and nutrition program. J Pediatr Hematol Oncol . 2009;31:259-266.

30. Malik VS, Pan A, Willett WC, Hu FB. Sugar-sweetened beverages and weight gain in children and adults: A systematic review and meta-analysis. Am J Clin Nutr . 2013;98:1084-1102.

31. Liu D, Zhao LY, Yu DM, et al. Dietary patterns and association with obesity of children aged 6-17 years in medium and small cities in China: Findings from the CNHS 2010-2012. Nutrients . 2018;11:3.

32. Spear BA, Barlow SE, Ervin C, et al. Recommendations for treatment of child and adolescent overweight and obesity. Pediatrics . 2007;120:S254-288.

33. Brand-Miller JC, Thomas M, Swan V, Ahmad ZI, Petocz P, Colagiuri S. Physiological validation of the concept of glycemic load in lean young adults. J Nutr . 2003;133:2728-2732.

34. Browne EK, Zhou Y, Chemaitilly W, et al. Changes in body mass index, height, and weight in children during and after therapy for acute lymphoblastic leukemia. Cancer . 2018;124:4248-4259.

35. Zhang FF, Rodday AM, Kelly MJ, et al. Predictors of being overweight or obese in survivors of pediatric acute lymphoblastic leukemia (ALL).Pediatr Blood Cancer . 2014;61:1263-1269.

36. Livingstone MB, Robson PJ, Wallace JM. Issues in dietary intake assessment of children and adolescents. Br J Nutr . 2004;92:S213-222.

37. Reilly JJ, Ventham JC, Newell J, Aitchison T, Wallace WH, Gibson BE. Risk factors for excess weight gain in children treated for acute lymphoblastic leukaemia. Int J Obes Relat Metab Disord . 2000;24:15371541.

\section{FIGURE LEGENDS}


FIGURE 1 Median BMI z-score by demographics and risk group at each timepoint Abbreviations: BMI, body mass index.

\section{Hosted file}

Low GI Main Table 1.pdf available at https://authorea.com/users/367476/articles/486880-abilingual-dietary-intervention-early-in-treatment-is-feasible-and-prevents-weight-gainin-childhood-acute-lymphoblastic-leukemia-all

\section{Hosted file}

Low GI Main Table 2.pdf available at https://authorea.com/users/367476/articles/486880-abilingual-dietary-intervention-early-in-treatment-is-feasible-and-prevents-weight-gainin-childhood-acute-lymphoblastic-leukemia-all

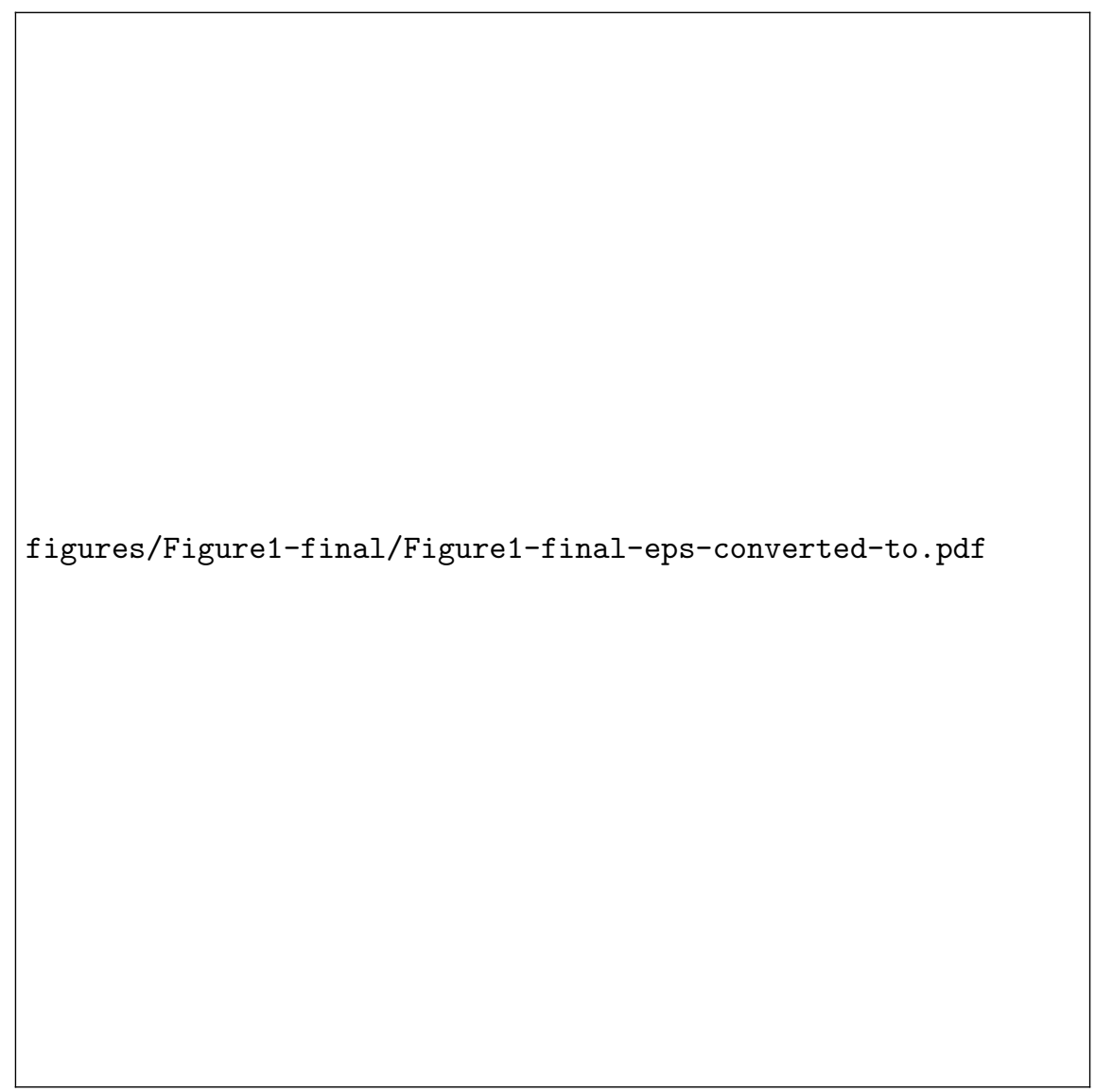

